\title{
Percepção dos alunos de medicina sobre a criação de um atlas fotográfico de anatomia humana com peças cadavéricas
}

\author{
Perception of medical students about the creation of a \\ photographic atlas of anatomy with cadaveric parts
}

\author{
Caroline Oliveira Nascimento', Camila Gomes de Souza', \\ Vivian de Oliveira Sousa Corrêa \\ 'Curso de Medicina, Universidade Federal do Rio de Janeiro, Campus UFRJ-Macaé, Macaé (RJ), Brasil.
}

\begin{abstract}
RESUMO
A compreensão da Anatomia Humana é base para interpretações corretas na prática profissional em saúde. Diferentes métodos para o aperfeiçoamento do ensino-aprendizagem vêm sendo introduzidos, dentre eles, a ferramenta digital é atrativa para os alunos e, aos poucos, vem sendo implantada. Objetivo: Obter a percepção dos acadêmicos sobre a incorporação de um atlas digital fotográfico retratando as peças do Anatômico do nosso Campus com o intuito de aprimorar o estudo prático dos alunos. Métodos: Peças do sistema osteoarticular foram fotografadas, editadas, apontando as estruturas anatômicas visíveis e organizadas por região. Disponibilizamos o atlas aos 25 discentes do primeiro período do curso de Medicina (2015-2). Um questionário foi aplicado para avaliar o uso desse método pelos acadêmicos. Resultados: 21 alunos afirmaram que utilizaram o atlas, desses, $68 \%$ acreditaram que este contribuiu muito para o entendimento. Em relação às vantagens da ferramenta, 17 acadêmicos destacaram a exposição de peças reais, 9 disseram que auxiliou na compreensão, enquanto 4 apontaram ter direcionado para a prova prática. Acerca dos aspectos negativos, 6 acadêmicos observaram a presença apenas do sistema osteoarticular e 2 criticaram a falta de estruturas anatômicas visíveis nas peças. Como sugestão para melhoria, 7 alunos propuseram a inserção dos demais sistemas. Conclusões: $O$ atlas auxiliou os acadêmicos positivamente nos seus estudos práticos estimulando a familiaridade com as peças e aumento da compreensão. A ferramenta apontada neste trabalho pode ser reproduzida por outras Instituições e pode ser desenvolvida e ampliada para utilização de recursos online, entre outros, de forma a facilitar o processo ensino-aprendizagem.
\end{abstract}

PALAVRAS-CHAVE: Anatomia. Educação. Aprendizagem. Atlas. Fotografia.

\section{COMO CITAR ESTE ARTIGO} Nascimento CO, Souza CG, Corrêa VOS. Percepção dos alunos de medicina sobre a criação de um atlas fotográfico de anatomia humana com peças cadavéricas. Interdisciplinary Journal of Health Education. 2017 Jul-Dez:2(2):81-88. https://doi.org/10.4322/ijhe.2017.001

\section{CORRESPONDÊNCIA}

Vivian de Oliveira Sousa Corrêa Universidade Federal do Rio de Janeiro Av. Aluizio da Silva Gomes, 50, Novo Cavaleiros, CEP 27930-560, Macaé (RJ), Brasil

sousa.vo@gmail.com

\section{FONTE DE FINANCIAMENTO} Próprio.

\section{CONFLITO DE INTERESSE}

Os autores declararam não

haver conflitos de interesse.

O estudo foi realizado na Universidade Federal do Rio de Janeiro - Campus UFRJ - Macaé, Macaé (RJ), Brasil.

Todos os autores leram e aprovam a versão final submetida ao Interdisciplinary Journal of Health Education (IJHE).

\section{ABSTRACT}

The understanding of Human Anatomy is the ground to corrects interpretations at the professional practice. Different methods to the improvement of teaching-learning have being introduced, which them, the digital instrument is attractive to academics, and little by little has being implanted. Objective: To obtain the perception of the academics about the incorporation of a photographic digital atlas portraying the pieces of the Anatomic lab of our Campus with the intention to improve the practical study of the students. Methods: Parts of the osteoarticular system were photographed, edited, pointed the anatomical structures visible and organized by region. It was available to the 25 medical students at first semester (2015-2). A questionnaire has applied to evaluate the use of this method by academics. Results: 21 students alleged that they used the photographic digital atlas, of which $68 \%$ believed that it contributed a lot to the understanding. Regarding the advantages of the tool, 17 academics highlighted the exhibition of real pieces, 9 said they helped in the comprehension, while 4 indicated that they had been directed to the practical test. About the negative aspects, 6 academics observed the presence of only the osteoarticular system and 2 criticized the lack of anatomical structures visible in the cadaverous parts. As a suggestion for improvement, 7 students proposed the insertion of the other systems. Conclusions: The atlas helped academics positively in their practical studies by stimulating familiarity with the pieces and increasing understanding. The tool pointed out in this work can be reproduced by other Institutions and can be developed and expanded to use online resources, among others, in order to facilitate the teaching-learning process. KEYWORDS: Anatomy. Education. Learning. Atlas. Photography. 


\section{Introdução}

O aprimoramento da formação médica no Brasil tem sido apontada como parte das ações para a melhoria do atendimento aos usuários do Sistema Único de Saúde (SUS). Em 2014, o Ministério da Educação aprovou as novas Diretrizes Curriculares Nacionais (DCNs) do curso de Medicina ${ }^{1}$, que especificam a formação de um médico proativo e crítico, capaz de atuar em diferentes níveis de atenção à saúde. Para isto, a aluno deve se corresponsabilizar pela sua formação desde o início da graduação e ainda se comprometer com a formação das próximas gerações de profissionais de saúde.

Anatomia Humana é uma disciplina de base para formação dos profissionais de saúde ${ }^{2,3}$, geralmente inserida desde o primeiro período nos cursos. O conteúdo da Anatomia inclui nomenclatura, forma e a localização das estruturas do corpo humano ${ }^{4}$, correlacionando-as com suas funções. Esta disciplina, tradicionalmente, utiliza acervos de peças cadavéricas como fonte central de conhecimento prático em todo o mundo. Desta forma, o aprendizado das estruturas no cadáver é importante para as correlações com o exercício futuro do profissional de saúde ${ }^{5}$.

Desde o início das práticas de estudo com o corpo humano existe a preocupação com o estado de conservação do cadáver ${ }^{5}$ e com os prejuízos a saúde que o contato prolongado com substâncias, como o formaldeído, podem causar' ${ }^{6}$. Soma-se o fato de que a reposição das peças cadavéricas, atualmente, está dificultada.

Com a reestruturação dos cursos de saúde para atender as $\mathrm{DCNs}^{1}$, os docentes tem buscado formas de estimular a autonomia e a proatividade do estudante. Atualmente, o acesso a ferramentas digitais está amplamente difundido, permitindo a inserção destas no meio acadêmico. Métodos que utilizam conteúdos digitais podem facilitar a aquisição do conhecimento nesta geração, além de permitir uma quebra do paradigma do professor como fonte principal do conhecimento ${ }^{7}$.

Com o incentivo à aquisição de conhecimento, por meio de metodologias ativas, recursos foram destinados aos laboratórios morfofuncional e de informática e biblioteca com o intuito de se valorizar o estudo autodirigido ${ }^{8}$. Estes métodos conduzem à melhoria de desempenho do aluno, facilitam o aprendizado de conteúdos considerados difíceis de compreender por meio de aulas expositivas e aumentam a correlação dos conteúdos ministrados com a prática clínica, permitindo a estruturação efetiva do conhecimento e impactando diretamente na formação crítica e qualidade profissional ${ }^{9}$.

Os métodos ativos de ensino-aprendizagem tem sido aplicados, mas não verificamos com frequência na literatura relatos sobre o uso de ferramentas para desenvolver o conteúdo prático ministrado nos cursos de saúde. A disciplina de Anatomia geralmente apresenta grande parte de sua carga horária de aulas práticas. No nosso Campus, por exemplo, as atividades práticas contabilizam cerca de $50 \%$ do conteúdo da disciplina nos diferentes cursos. Durante o estudo prático da Anatomia, os alunos precisam correlacionar as imagens dos atlas comercializados com as peças cadavéricas reais no Laboratório, que não é uma tarefa trivial. Desta forma, esta disciplina tem o desafio de desenvolver atividades práticas que auxiliem os alunos no processo de construção do conhecimento num universo de conteúdos ministrados.

Este trabalho teve como objetivo obter a percepção dos acadêmicos sobre a introdução de um novo método digital no estudo prático: um atlas contendo fotografias de peças cadavéricas do Laboratório de Anatomia do Campus. Desenvolvemos esse atlas com informações descritivas dos acidentes anatômicos presentes nas amostras, incentivando o aluno a adquirir conhecimento ativamente através do estudo dirigido por esta ferramenta. 


\section{Métodos}

Desenho do estudo e população

Foi realizado um estudo descritivo, de corte transversal, em que foram avaliados estudantes de um curso de Medicina, na disciplina de Anatomia do Aparelho Locomotor, ministrada no primeiro semestre. Foram incluídos os 25 estudantes da turma de 2015-2, que, após explicação sobre a pesquisa, decidiram espontaneamente participar e assinaram o termo de consentimento livre e esclarecido, conforme aprovação por comitê de ética (CAAE: 47816915.4.0000.5291).

\section{Confecção do atlas de Anatomia com peças cadavéricas}

Para o desenvolvimento do Atlas de Anatomia Humana, as peças cadavéricas do sistema osteoarticular do Laboratório de Anatomia do Campus foram selecionadas tendo como critério a preservação das estruturas anatômicas. Estas peças foram fotografadas em diferentes ângulos utilizando uma câmera digital. Um dos grandes diferenciais desse atlas são as diferentes vistas das peças fugindo do que é apresentado em atlas convencionais. As imagens foram transferidas para um programa de edição de imagens, onde foram analisadas e editadas. Após a seleção das melhores imagens de cada peça, as estruturas anatômicas foram apontadas e descritas utilizando como referência livros-texto ${ }^{10,11}$ e atlas de Anatomia conceituados ${ }^{12,13}$. As imagens foram agrupadas e organizadas de acordo com a topografia, sendo dividido em módulos: esqueleto axial, apendicular superior e inferior (compreendendo as regiões de cíngulo e os membros) (Figura 1). Os módulos de esqueleto axial e apendicular superior foram disponibilizados para os 25 discentes do primeiro período (2015-2) do curso de Medicina do Campus através da internet.

\section{Coleta e análise dos dados}

O instrumento foi confeccionado, validado e aplicado aos acadêmicos ao final das atividades. As respostas foram acompanhadas por assinatura de Termo de Consentimento Livre e Esclarecido para uso das informações. O questionário continha quatro perguntas objetivas em que se abordava: a utilização desse atlas, o quanto contribuiu para a compreensão da disciplina, se fez uso de outro atlas de Anatomia e qual método de estudo foi o de preferência para os acadêmicos. Este questionário também continha três questões subjetivas relacionadas às vantagens, desvantagens e sugestões para o uso do Atlas de Anatomia. Os dados foram analisados no programa Microsoft Excel.

\section{Resultados e discussão}

Entre os acadêmicos que participaram da pesquisa, 21 (84\%) utilizaram o atlas confecionado em seus estudos e quatro (16\%) afirmaram não terem utilizado. A boa adesão ao atlas corrobora o fato de que, nas últimas décadas, observou-se o aumento das inovações tecnológicas e os indivíduos nascidos entre 1982 e 2002 tiveram uma mudança nos hábitos de vida diários e nas formas de aprendizagem ${ }^{14}$. Assim, cada vez mais o método tradicional de ensino vem sendo questionado, sendo necessária a introdução de novas abordagens nas disciplinas, e as ferramentas digitais são opções interessantes para esta geração.

Os instrumentos tecnológicos são variados e dinamizaram o aprendizado da Anatomia, como notebook, discos laser, fibras óticas, dispositivos de multimídia e videodiscos. Estes proporcionaram a transformação das bibliotecas tradicionais em midiatecas com a reunião de acervos diversificados ${ }^{15}$, aumentando o contato dos indivíduos com diversos conhecimentos. Essas ferramentas, como o atlas implementado neste trabalho, contribuem para a habilidade de visualizar objetos tridimensionais sob diferentes ângulos permitindo a identificação das estruturas anatômicas, o que é imprescindível para o estudantes da área da saúde ${ }^{16}$. 


\section{ijhe}

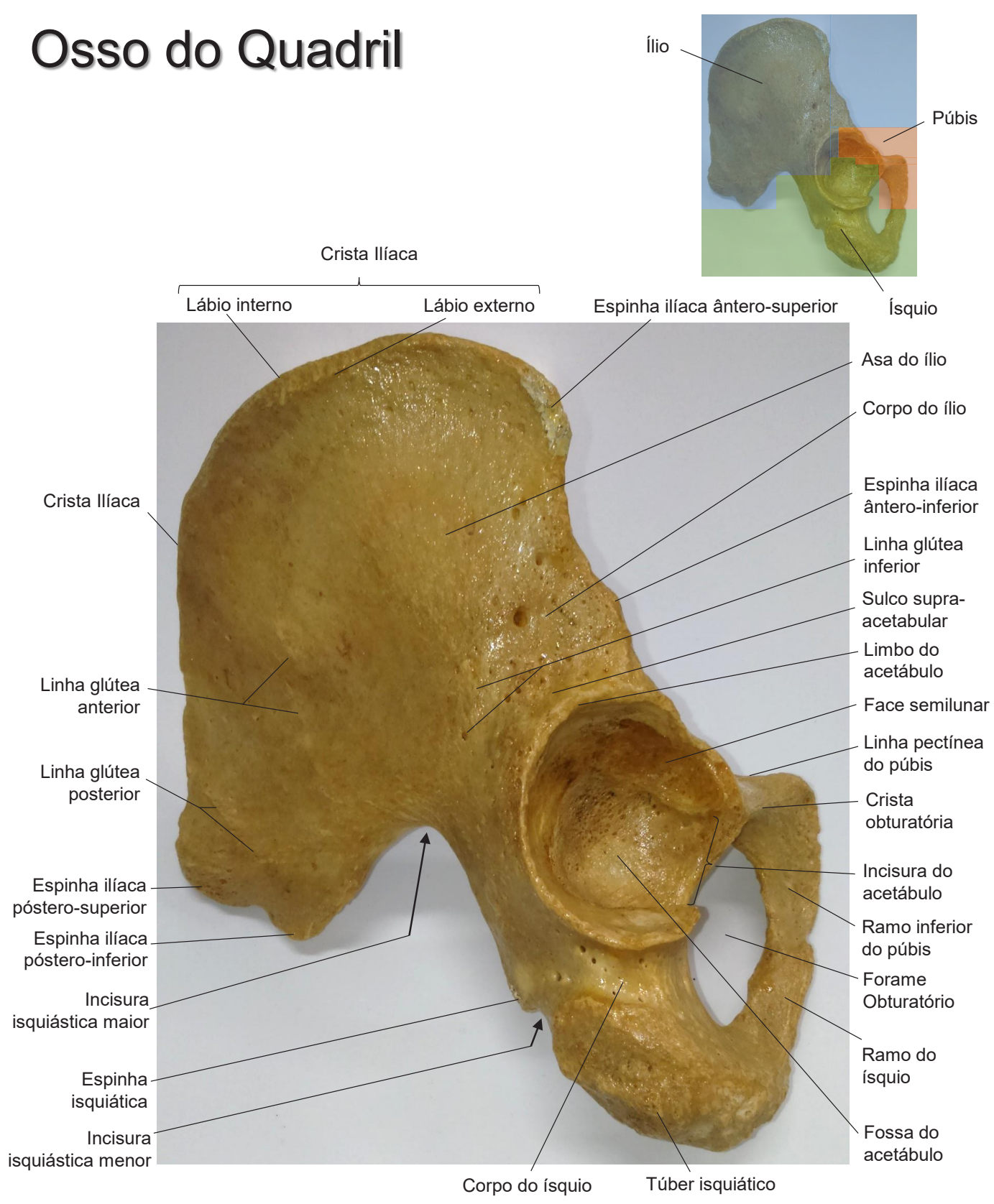

Ilíaco direito; vista lateral.

Figura 1. Página do atlas fotográfico. Página do atlas que contém o osso ilíaco, pertencente ao cíngulo do membro inferior, em vista lateral. A imagem ressalta os acidentes anatômicos presentes na peça cadavérica.

Dos alunos que usaram o atlas, 17 (68\%) assinalaram que esse contribuiu "muito" para a compreensão sobre Anatomia, enquanto três (12\%) que "pouco" contribuiu, seis $(24 \%)$ não responderam e a opção "nada" não foi escolhida por nenhum dos acadêmicos. Sabe-se que diferentes estímulos auxiliam na consolidação do aprendizado teórico/prático. A soma dos sentidos visão, audição e tato é responsável pela taxa de retenção de informação de cerca de $70 \%$. Esta taxa está relacionada a outros fatores, como motivação ${ }^{17}$. A utilização dessa ferramenta visual auxilia a fixação do conteúdo estudado em Anatomia. O aluno que participa das aulas práticas, escuta as demonstrações, pega as peças para estudar e ainda utiliza o atlas fotográfico, pode ter grande aproveitamento na retenção do conteúdo de Anatomia. Além disso, o uso 


\section{ijhe}

da ferramenta sem o auxílio do professor presencialmente constitui um estímulo para o aprendizado ativo.

Todos os discentes que usaram o atlas do projeto afirmaram utilizar outro atlas de Anatomia durante os seus estudos. Em relação à preferência, 20 (80\%) optaram por ambos os métodos o fotográfico e o convencional, quatro (16\%) abstiveram-se, um (4\%) preferiu usar apenas o atlas confeccionado e nenhum acadêmico preferiu utilizar apenas o atlas convencional. No primeiro período do curso de Medicina, a Anatomia do Aparelho Locomotor é ministrada de forma regional (abrangendo os ossos, grupos musculares, articulações, inervações e vascularizações), enquanto o atlas fotográfico criado continha apenas ossos e articulações. Com isso, o uso concomitante de outros atlas foi necessário para complementação do conteúdo abordado. Ainda assim, a maioria prefere utilizar também o atlas com peças do laboratório de Anatomia.

Um trabalho semelhante foi introduzido no Departamento de Anatomia da Universidade Estadual de Londrina (UEL), onde um software com atlas fotográfico apresentando os sistemas osteoarticular e muscular permitiram o auxilio e dinamização nas aulas e estudos da Anatomia ${ }^{18}$. Outro exemplo, na Pontifícia Universidade Católica do Chile utilizou métodos computadorizados de aprendizagem na abordagem da Anatomia e constatou relatos positivos em sua utilização, como a facilidade em compreensão dos conteúdos (destaque para os complexos), além de atuarem como uma ferramenta motivacional no estudo tradicional da Anatomia Humana ${ }^{19}$.

Como vantagens do atlas fotográfico citadas pelos alunos temos (Figura 2): $17(68 \%)$ pontuaram a utilização de peças reais, outras $9(36 \%)$ destacaram maior compreensão, quatro $(16 \%)$ apontaram o direcionamento para a prova prática, um (4\%) a facilidade de acesso, um (4\%) a boa organização e boa elaboração e quatro (16\%) não responderam à pergunta. As vantagens mostram que os acadêmicos tiveram uma
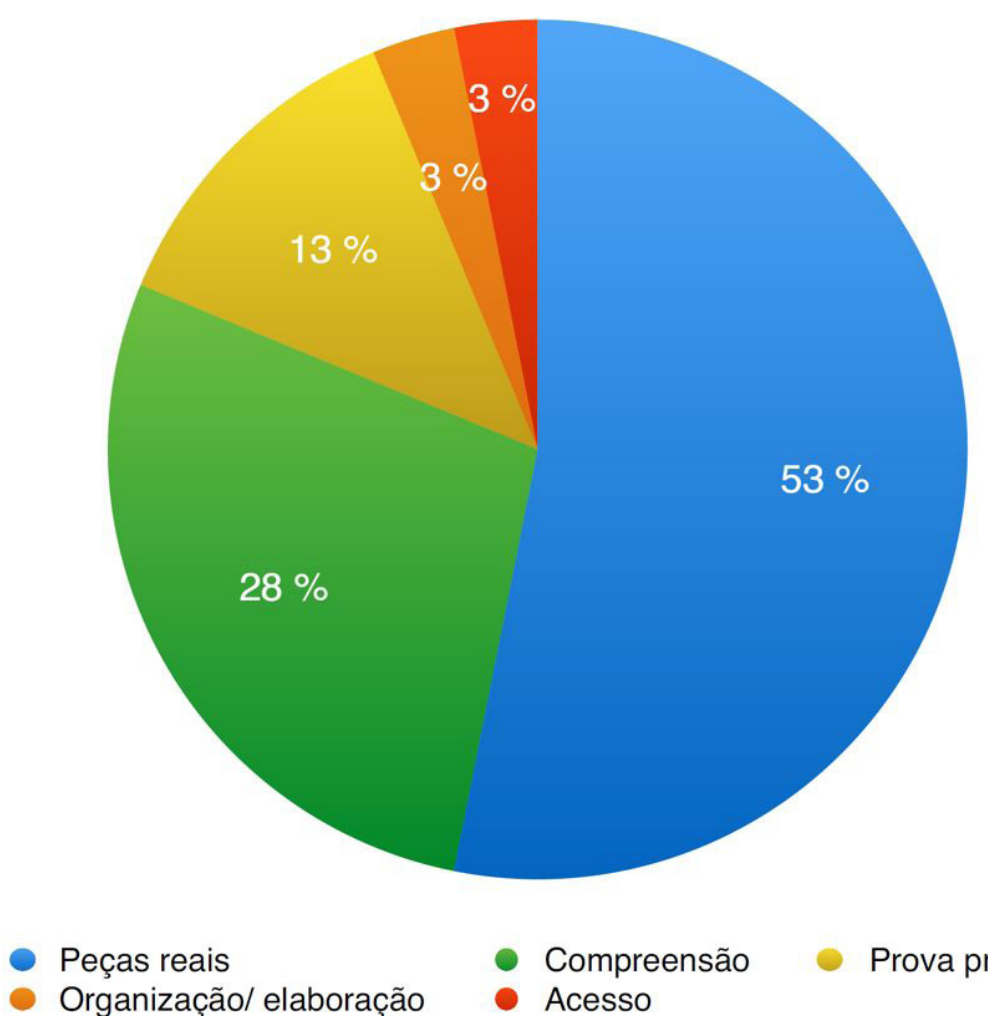

- Compreensão
Acesso

Prova prática

Figura 2. Vantagens do atlas fotográfico pontuadas pelos estudantes, organizadas a partir de palavras-chave. A maioria dos alunos relata a vantagem do uso de peças reais (azul, 53\%), o que facilita o estudo e, consequentemente, melhora a compreensão (verde, 28\%). Alguns relacionaram o fato de auxiliar o estudo para a avaliação prática (amarelo, 13\%). Foi citado a organização e elaboração do atlas como um ponto positivo (laranja, 3\%) e a facilidade ao acesso (vermelho, 3\%), visto que atlas de Anatomia são geralmente caros e nosso atlas foi disponibilizado sem custo para os alunos. 


\section{ijhe}

maior familiaridade utilizando as fotografias das peças do Anatômico, o que pode ter proporcionado um maior entendimento e preparação para as avaliações práticas.

Sobre as desvantagens do atlas confeccionado (Figura 3), seis (24\%) relataram apenas a presença do sistema osteoarticular, três (12\%) não destacaram desvantagens, duas ( $8 \%$ ) afirmaram a falta de estruturas nas peças, um (4\%) questionou a quantidade limitada de imagens, um (4\%) apontou a não disponibilização de todos os módulos do atlas, um (4\%) citou a veiculação do atlas apenas digital e 12 (48\%) não responderam. Quanto a restrição de apenas a apresentação do sistema osteoarticular, percebe-se o interesse dos alunos na abrangência dos outros sistemas, dessa forma concluímos que a metodologia empregada foi positiva no entendimento dos acadêmicos, representando uma vantagem para o trabalho exposto. Como, inicialmente, o projeto visava apresentar esse sistema, a sua extensão a outros sistemas estava condicionada à aceitabilidade dos acadêmicos. Já a falta de estruturas nas peças é explicada pelo intenso desgaste dos órgãos cadavéricos devido ao amplo uso por anos e pela grande utilização nos cursos da área biomédica, isso associado a dificuldade de reposição atual dessas peças. Portanto, o armazenamento através do registro digital, se torna uma ferramenta importante, também, para manter de alguma forma um acervo.

Na Figura 4, são apresentadas as sugestões: sete (28\%) propuseram a inserção de outros sistemas, um (4\%) opinou pela adição de imagens radiológicas, um (4\%) recomendou mais imagens das estruturas, um (4\%) aconselhou a disponibilização de todos os módulos do atlas, um (4\%) não possui sugestão e 15 (60\%) não responderam. Devido a boa aceitação do atlas, este já está em processo de expansão para inclusão de todos os sistemas, atendendo a todos os cursos que abordam Anatomia Humana, que inclui não só a Medicina, mas também Enfermagem e Obstetrícia, Nutrição, Farmácia e Ciências Biológicas.
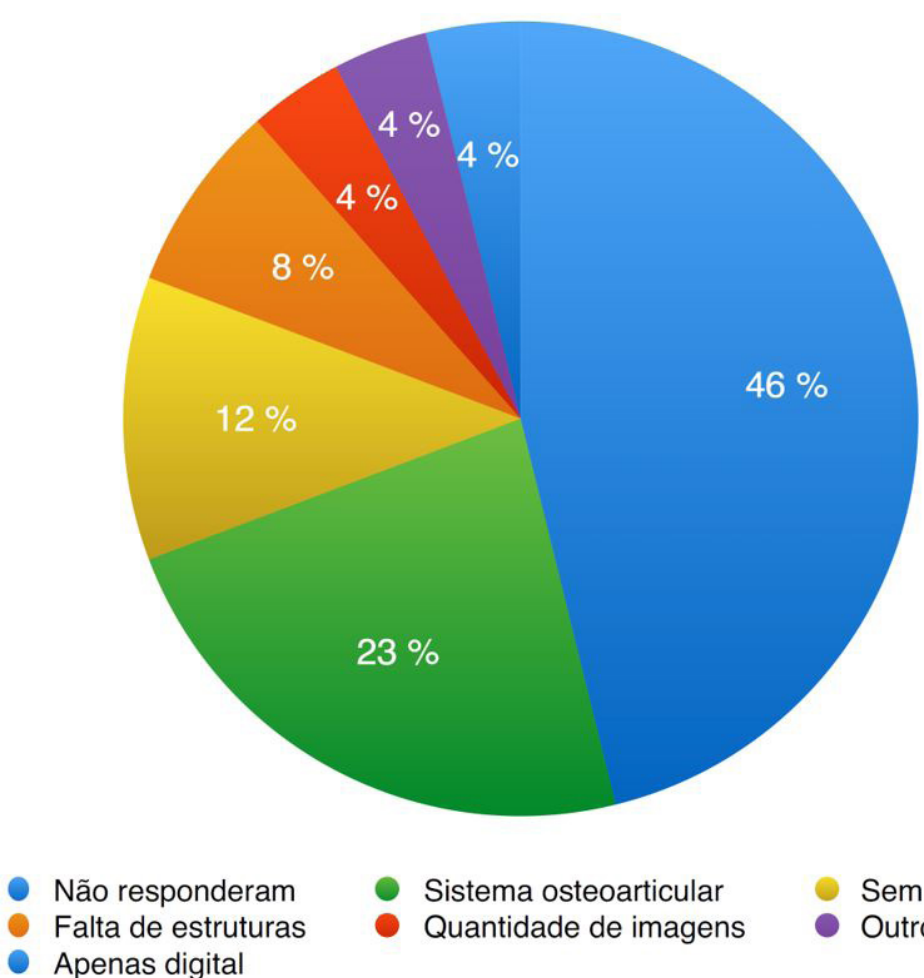

Sistema osteoarticular

Quantidade de imagens
Sem desvantagem

Outros módulos

Figura 3. Desvantagens apresentadas pelos alunos, organizadas em expressões-chave de maior prevalência. Grande parte dos alunos não relatou nenhuma desvantagem do atlas fotográfico (azul, 46\%) e alguns escreveram que não enxergavam desvantagens (amarelo, 12\%). Quase um quarto dos alunos citou a presença somente do sistema ostoarticular (verde, $23 \%$ ). Alguns sentiram falta de algumas estruturas (laranja, 8\%). E foi citado que havia um número pequeno de imagens (vermelho, 4\%), a ausência do uso do atlas em outros módulos de Anatomia (roxo, 4\%) e o fato de o atlas ser apensa digital (azul, 4\%). 


\section{ighe}

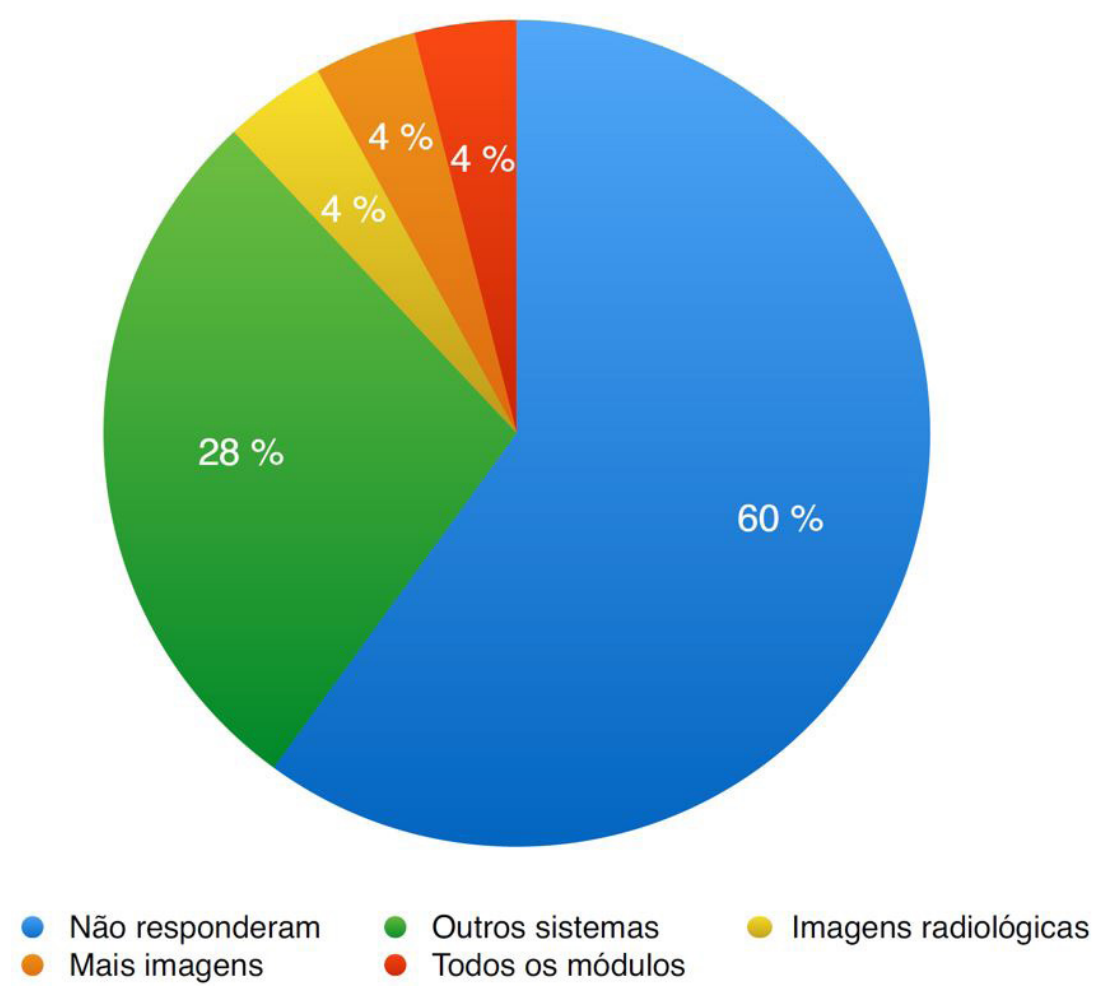

Figura 4. Sugestões apontada pelos acadêmicos, quanto a melhoria do Atlas fotográfico. As sugestões apontadas pelos alunos incluem: inclusão de outros sistemas anatômicos (verde, 28\%), incluir imagens radiológicas (amarelo, 4\%) aumentar o número de imagens (laranja, 4\%) e incluir o uso do atlas em todos os módulos onde a disciplina Anatomia Humana é estudada. A maioria (azul, 60\%) não deixou sugestão.

\section{Conclusões}

O atlas fotográfico de Anatomia Humana contendo as peças cadavéricas do sistema osteoarticular do Anatômico do nosso Campus constituiu uma ferramenta de auxílio para o estudo prático de acordo com a percepção dos alunos de Medicina no primeiro período. Verificamos neste trabalho, que o atlas foi aceito pelos alunos, contribuindo para o aumento da familiaridade dos acadêmicos com peças cadavéricas humanas e potencializando a compreensão das estruturas abordadas. Além do uso pelos alunos, o registro das estruturas é de grande valia para formar um acervo de dados, visto que as peças são naturalmente desgastadas com o uso por diferentes turmas de cursos variados ao longo de tempo.

Este trabalho tem um importante impacto socioeconômico, uma vez que a chegada na Universidade gera gastos e muitos alunos não tem possibilidade de adquirir um atlas no primeiro período. Muitos Campi que foram implantados, resultados da interiorização, ainda não possuem bibliotecas equipadas de forma a atender a demanda dos estudantes e, tão pouco, moradia (como alojamentos) e locais para alimentação dos estudantes a preços reduzidos, tudo isso aumenta os custos para se manter na Universidade. A produção de uma ferramenta tão útil para o processo de ensino-aprendizagem e que ainda não tem custo para os alunos possui grande préstimo às Instituições de Ensino Superior.

Concluímos que o atlas fotográfico com peças cadavéricas pode ser ampliado para utilização por outros cursos de saúde, inclusão dos diversos sistemas (conforme solicitado pelos alunos), associação com recursos on line, entre outros, com o fim de promover o ensino-aprendizagem do acadêmico, sempre colocando este como protagonista dos seus estudos nessa disciplina que é tão importante na prática dos cursos de saúde e incentivando o "aprender a aprender". 


\section{Referências}

1. Brasil. Ministério da Educação. Resolução n ${ }^{\circ} 3$, de 20 de junho de 2014. Institui Diretrizes Curriculares Nacionais do Curso de Graduação em Medicina e dá outras providências. Diário Oficial da União, DF, Brasília, 20 jun. 2014.

2. Siqueiro EGB No, Ferreira JR. O ensino da anatomia humana no curso de medicina da Universidade Federal de Goiás avaliação e perspectivas. Arq Ciênc Saúde UNIPAR. 2001;5(1):41-50.

3. Kruse MHL. Anatomia: a ordem do corpo. Rev Bras Enferm. 2004;57(1):79-84. http://dx.doi.org/10.1590/S003471672004000100017. PMid:15473437.

4. Braz PRP. Método didático aplicado ao ensino da anatomia humana. Anuário da Produção Acadêmica Docente. 2009; 3(4):303-10.

5. Kimura AK, Carvalho WL. Estudo da relação custo $x$ benefício no emprego da técnica de glicerinação em comparação com a utilização da conservação por formol [Trabalho de conclusão de curso]. Araraquara: Universidade Estadual Paulista.

6. Veronez DAL, Farias ELP, Fraga R, Freitas RS, Petersen ML, Silviera JRP. Potencial risco para a saúde ocupacional de docentes, pesquisadores e técnicos de anatomia expostos ao formaldeído. InterfacEHS [Internet]. 2006 [citado em 2017 Nov 29];1-14. Disponível em: http://www3.sp.senac.br/hotsites/blogs/InterfacEHS/wp-content/uploads/2013/08/4_ARTIGO_vol5n2. pdf

7. Limberger JB. Metodologias ativas de ensino e aprendizagem para educação farmacêutica: um relato de experiência. Interface Comunic Saúde Educ. 2013;17(47):969-75. http://dx.doi.org/10.1590/1807-57622013.3683.

8. Gomes R, Francisco AM, Tonhom SFR, Costa MCG, Hamamoto CG, Pinheiro OL, et al. A formação médica ancorada na aprendizagem baseada em problema: uma avaliação qualitativa. Interface Comunic Saúde Educ. 2009;13(28):71-83. http:// dx.doi.org/10.1590/S1414-32832009000100007.

9. Mota MF, Mata FR, Aversi-Ferreira TA. Constructivist pedagogic method used in the teaching of human anatomy. Int J Morphol. 2010;28(2):369-74. http://dx.doi.org/10.4067/S0717-95022010000200005.

10. Standring S. Gray’s: anatomia. 40. ed. Rio de Janeiro: Elsevier; 2010.

11. Moore KL. Anatomia Orientada para a Clínica. 7. ed. Rio de Janeiro: Guanabara Koogan; 2014.

12. Paulsen F, Waschke J. Bobotta: Atlas de Anatomia. 23. ed. Rio de Janeiro: Guanabara Koogan; 2012.

13. Netter FH. Atlas de Anatomia Humana. 5. ed. Rio de Janeiro: Elsevier; 2011.

14. Sohrabi B, Iraj H. Implementing flipped classroom using digital media: a comparison of two demographically different groups perceptions. Comput Human Behav. 2016;60:514-24. http://dx.doi.org/10.1016/j.chb.2016.02.056.

15. Fornaziero CC, Gil CRR. Novas tecnologias aplicadas ao ensino da anatomia humana. Rev Bras Educ Med. 2003;27(2):141-6.

16. Azer SA, Azer S. 3D anatomy models and impact on learning: a review of the quality of the literature. Health Prof Educ. 2016;2(2):80-98. http://dx.doi.org/10.1016/j.hpe.2016.05.002.

17. Davis BT. Introduction to multimedia in museums [Internet]. 1996 [citado em 2017 Mar 5]. Disponível em: http://www. emuzeum.cz/admin/clanky/files/280-introtomultimediamuseums.pdf

18. Yoshida M, Medeiros DA Fo, Saito DS, Araújo JC, Fornaziero CC, Omoto MHH. LocomoShow - uma ferramenta de apoio ao ensino da anatomia humana [Internet]. Belo Horizonte: UFMG; 2003 [citado em 2017 Nov 29]. 9 p. Disponível em: http://www.lbd.dcc.ufmg.br/colecoes/wim/2003/007.pdf

19. Guiraldes del Canto H, Oddó Atria H, Ortega FX, Oyarzo MP. Metodos computacionales y graficos de apoyo al aprendizaje de la anatomia humana (vision de los estudiantes). Rev Chil Anat. 1995;13(1):67-71.

\section{Contribuição dos autores}

Caroline Oliveira Nascimento contribuiu com a coleta e tratamento dos dados, análise e interpretação, levantamento da literatura, redação e revisão crítica. Camila Gomes de Souza contribuiu com a coleta e tratamento dos dados, análise e interpretação, levantamento da literatura, redação e revisão crítica. Vivian de Oliveira Sousa Corrêa participou na concepção e desenvolvimento, desenho metodológico, supervisão, coleta e tratamento dos dados, análise e interpretação, levantamento da literatura, redação e revisão crítica. 